\title{
Methylene Blue: Its Efficacy and Safety as a Storage Solution in Hair Transplantation
}

\author{
Chinmanat Tangjaturonrusamee, MD, Patcharee Thientaworn, RN, Natenapa Arunrattanapong, RN, \\ Dell Kristie O. Castillejos, MD, Damkerng Pathomvanich, MD, FACS Bangkok, Thailand path_d@hotmail.com
}

\section{Introduction}

Successful optimal outcome of hair transplantation is associated with several factors. Factors affecting poor growth include desiccation, trauma from manipulation and implantation, quality of the follicular units (FUs), duration of the FUs between harvesting and implantation, perfusion of the recipient site, and post-operative care.

With respect to preservation of the FU grafts, for the past decades, normal saline and lactated Ringer's solution have provided acceptable results compared to higher priced solutions such as HypoThermosol, ATPv, platelet rich plasma (PRP), and PlasmaLyte A. However, there are no other inexpensive solutions that have the potential benefits in storage solution. This prompts us to investigate methylene blue (MB) as an additive antioxidant to normal saline for storage solution and it is widely available at low cost.

$\mathrm{MB}$ is used primarily as a dye and reducing agent for the treatment of methemoglobinemia. However, in theory, MB additionally has known energy enhancing and antioxidant properties. During hair transplantation surgery, biochemical injury occurs when hair follicles are transferred from an ischemic phase after harvesting to a reperfusion phase from blood oxygenation after implantation. ${ }^{1}$ This ischemia-reperfusion injury leads to reactive oxygen species, therefore damaging cells, and thereby inducing suboptimal growth as a result. ${ }^{1,2}$ Reported studies have shown that $\mathrm{MB}$ competitively inhibits the reduction of reactive oxygen species and helps to prevent adhesion. ${ }^{3,4}$

Recently, MB has been used as a treatment for refractory distributive shock from a variety of causes, such as sepsis and anaphylaxis, through a lesser-known property-inhibition of the downstream effect of nitric oxide. ${ }^{5}$ By inhibiting nitric oxide synthase and guanylate cyclase, MB has been associated with improved vascular reaction in post-reperfusion syndrome during liver transplantation (Figure 1). ${ }^{6} \mathrm{MB}$ has an important role in peri-operative vasoplegia refractory to vasopressin and catecholamine therapy in cardiac and liver surgery. ${ }^{6}$

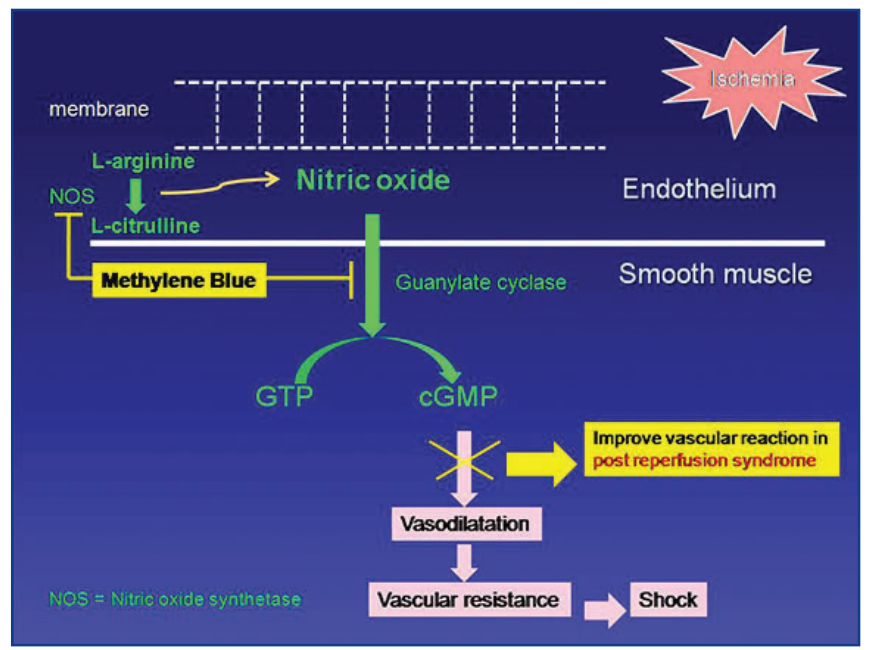

Figure 1. Representation of the inhibition of nitric oxide pathway by MB, improving postreperfusion syndrome.
In addition, MB increases ATP content and improves survival of skin fibroblast in Leigh Syndrome French Canadian (LSFC), which is a recessive disease caused by mutations in the LRPPRC gene (leucine-rich pentatricopeptide repeat containing protein). ${ }^{7}$ These mutations induce a cytochrome c oxidase (COX) deficiency resulting in episodes of acute acidotic crisis that will often lead to death. MB is a redox dye that increases COX content and activity in vitro and in vivo suggesting that $\mathrm{MB}$ could prevent and treat LSFC. ${ }^{7}$

The use of MB seems to be an effective antioxidant. However, the effect of MB on oxidative stress is controversial. The antioxidant effect of MB appears to be dose-dependent and associated, at least in part, upon the differences in organ systems and mode of application. ${ }^{4}$ To find whether MB could be used safely and effectively as a storage solution during hair transplantation surgery, and since no previous studies had been published regarding the use of a MB solution as a hair transplant storage solution, a study was undertaken to investigate MB's applicability, efficacy, and safety during hair transplant procedures, as well as to establish the optimal MB parameters for such a storage solution.

\section{Objective}

The objective of this study was to evaluate the efficacy and safety of methylene blue as a storage solution for hair growth enhancement in hair transplantation.

\section{Material and Methods}

This is an ISHRS research grant funded in October 2014.

\section{Patient Population}

Three subjects 18 years of age or older with male pattern hair loss (Norwood-Hamilton Class IV-VI) were chosen for this study. Patients were excluded if they 1) had underlying unstable medical conditions, 2) received topical medication for the hair loss within 6 months, 3) took finasteride or dutasteride or oral minoxidil within 12 months, 4) received light or laser therapy for the hair loss within 3 months, 5) received any medications that could cause hypotrichosis or hypertrichosis, 6) received any other treatments that could affect hair growth, or 7) were allergic to MB.

\section{Study Design}

This was a pilot study. All subjects gave their informed consent before entering the study. The subjects were required to participate in eight post-surgical evaluations during the course of the study — on the surgery date and seven follow-ups at 1 day, 7 days, 1 month, 4 months, 8 months, 12 months, and 18 months. Before surgery, two rows of nine separate $1.1-1.3 \mathrm{~cm} \times 1.1-1.3 \mathrm{~cm}$ study boxes were marked at the end of hairline on top of the forehead. The corners of each box were tattooed and a $1.2 \mathrm{~mm}$-wide bald skin area was left around each box. Boxes were designed for 20-35 grafts per box from four different types of storage solutions. In each row, the center box was used for grafts prepared 
in normal saline solution (NSS), while the boxes on either side outward were for grafts prepared in 4 different strengths of $\mathrm{MB} / \mathrm{NSS}$ in the order of $1,2,4$, and 8 drops, respectively (Figure 2).

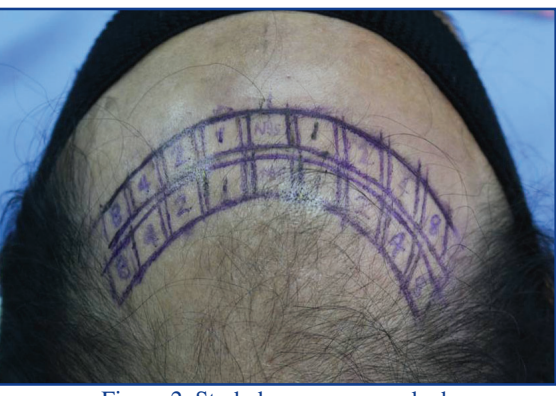

Figure 2. Study boxes were marked.

All three patients

underwent hair transplantation using the FUT technique. Before grafts were implanted, they were stored in four different strength solutions of MB in NSS and also in normal saline as a control to be surgically implanted in the center boxes. Two different concentrations of MB stock solutions were used to prepare the MB storage solutions utilized in this study. In the first patient, a $1 \%$ MB in NSS stock solution was utilized in preparing the four different strength storage solutions, while in the other two patients, a $0.1 \% \mathrm{MB}$ in NSS stock solution was utilized for preparing the different storage solutions. To make the four different strength MB storage solutions, 1, 2, 4, and 8 drops of the respective MB stock solution was added to each graft bowl containing $10 \mathrm{ml}$ NSS. All grafts were stored at $40^{\circ} \mathrm{F}\left(4.4^{\circ} \mathrm{C}\right)$ until being surgically transplanted into the patient's scalp.

The 1-hair FU grafts were placed in the front (outside) row, and the 2-hair FU grafts were placed in the back (next to hairline) row with $0.8 \mathrm{~mm}$ and $1 \mathrm{~mm}$ slit sites, respectively, by an experienced technician. Additionally, in the third patient, 3-hair FU grafts prepped with four different strengths of MB in NSS storage solutions were placed in five extra boxed random locations on the scalp. We did the 3-hair graft box study only in the third patient since the other two patients did not have enough 3-hair grafts.

\section{Efficacy Evaluation}

Photographs were taken of each box and hair counts were estimated at 4, 8, 12, and 18 months. Hair counts were performed by two independent technicians. Miniaturized hairs were not included in the hair counts.

\section{Safety Evaluation}

Subjects were evaluated post surgically on the first day and then again at 7 days, 1 month, 4 months, 8 months, 12 months, and 18 months. Adverse events such as skin irritation and discoloration of the skin or body fluid were recorded throughout the study.

\section{Results}

All three subjects were Thai males, ages 31, 40, and 47 years old, with pattern hair loss (Norwood-Hamilton Class IV-VI). Figures 3-6 represent the percentage of hair growth at 4, 8, 12, and 18 months, respectively, compared to the number of hairs at baseline. At post-op month 4, the survived grafts in the NSS boxes were superior to those in the MB boxes of all concentrations except for 3-hair grafts. However, at post-op months 8, 12, and 18, graft survival in the NSS boxes was superior to that in the MB boxes in all categories (Figure 7).

Figure 8 shows the graft survival in different drops of $\mathrm{MB}$ and it is clear that MB at a low concentration was better than MB at a higher concentration.

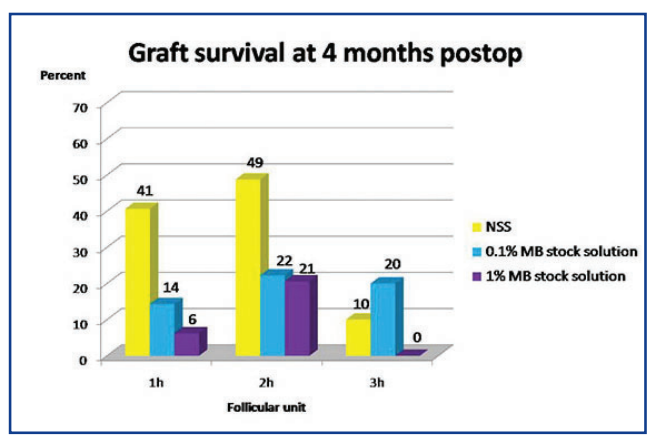

Figure 3. Comparison of post-op graft survival at 4 months.

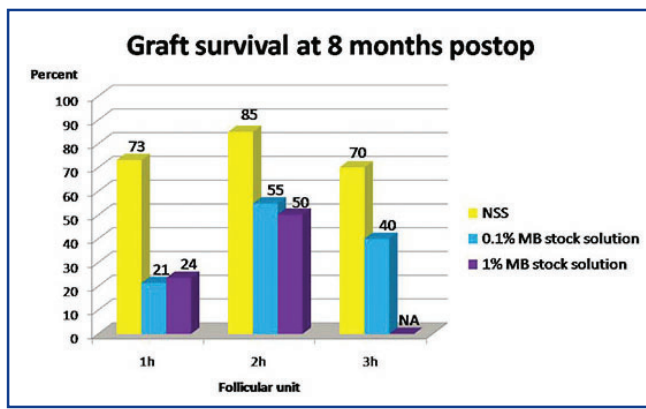

Figure 4. Comparison of post-op graft survival at 8 months.

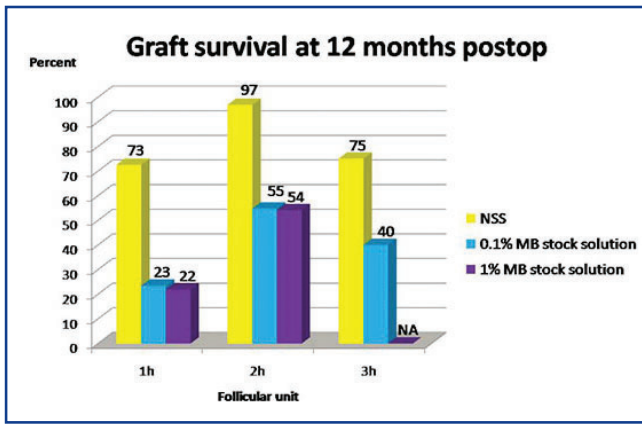

Figure 5. Comparison of post-op graft survival at 12 months.

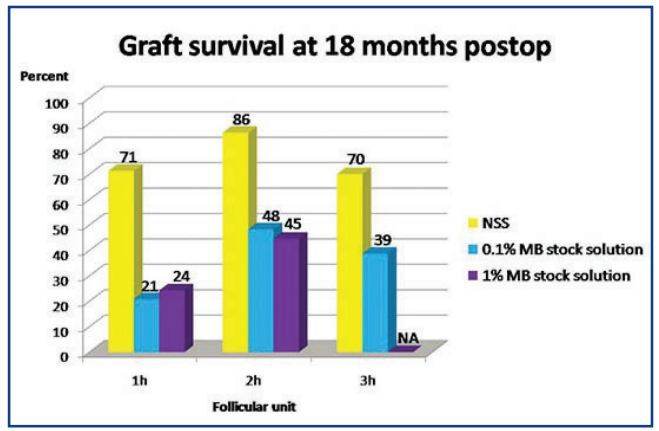

Figure 6. Comparison of post-op graft survival at 18 months.

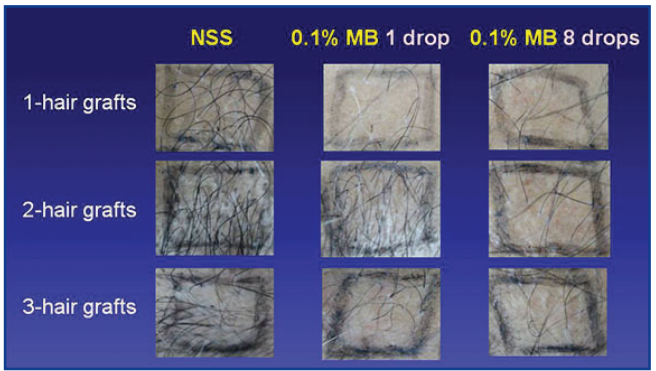

Figure 7. The post-op graft survival of NSS was superior to MB at 8 months.

No skin irritation or discolorations (including color changes of body fluid) were observed in those subjects.

\section{Discussion}

There are only a few storage solutions available worldwide that are inexpensive and yet effective for hair graft growth, such as normal saline and lactated Ringer's solution. There is no

$\Rightarrow$ page 196 
Methylene Blue from page 195

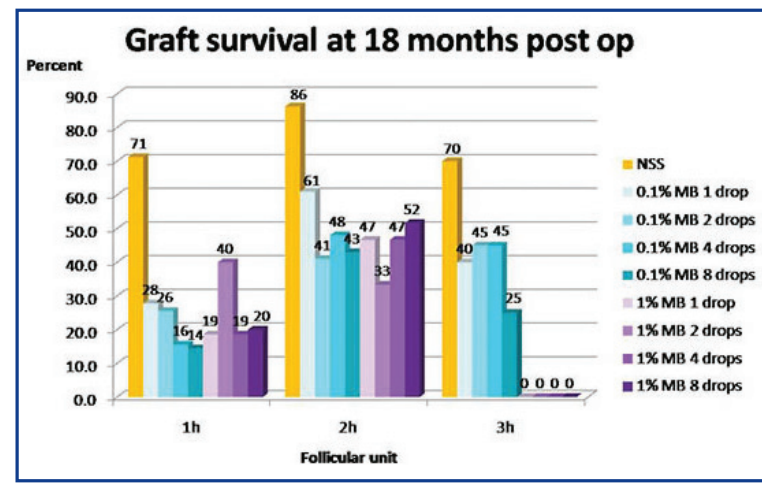

Figure 8. Comparison of post-op graft survival of MB in different concentrations at 18 months.

previously reported study of MB used in such cases. This study showed the efficacy and safety of MB in different strength storage solutions during hair transplantation surgery.

MB's antioxidant properties reduce reactive oxygen species in ischemia-reperfusion injury during the surgery. Unfortunately, our findings provide evidence that MB's efficacy to increase the survival rate of FU grafts is lower than that of NSS. At postop follow-up at 4 months, hair counts showed varied results among the three patients. The results demonstrated that NSS was superior to $\mathrm{MB}$ in hair growth except in the 3-hair FU grafts. However, the results at post-operative months 8,12 , and 18 indicated that NSS was superior to the MB storage solution involving hair growth of all FU grafts. The hair growth rate of $0.1 \%$ and $1 \% \mathrm{MB}$ stock solution were compatible. These findings provide evidence that MB's efficacy to increase the growth rate of FU grafts is not better than NSS as a storage solution. The experimental findings are suggestive that the antioxidant properties of MB do not improve the survival of hair grafts, but rather decrease it.

It is debatable among hair transplant surgeons whether MB may cause follicular toxicity since some hair transplant surgeons use MB to mark the recipient site or to dye grey hair during the slivering and cutting of grafts; nevertheless, they did not experience the poor hair growth rate. In our opinion, this could be explained by the length of contact time between $\mathrm{MB}$ and hair grafts. The contact time with FU grafts when using MB as a storage solution is longer than when it is used as a dye in the slivering and cutting process or as a marker in recipient site preparation, and this might affect graft survival.

While this study consisted of a small sample size, it nevertheless has produced new and clinically important data supporting positive strategies regarding the application of MB storage solutions for enhancing hair growth rate in hair transplantation.

\section{Conclusion}

Methylene blue at all concentrations as discussed appears to decrease graft survival in hair transplantation in this limited pilot study. It has proven to be less effective than NSS as the sole storage solution. However, additional studies should be performed with lower and different concentrations of MB to confirm its efficacy as a potential storage solution.

\section{References}

1. Cooley, J.E. Ischemia-reperfusion injury and graft storage solutions. Hair Transplant Forum Int'l. 2004; 14(4):121-39.

2. Crisostomo, M.R., et al. Oxidative stress in follicular units during hair transplantation surgery. Aesthetic Plast Surg. 2011(Feb); 35(1):19-23.

3. Salaris, S.C., C.F. Babbs, and W.D. Voorhees. Methylene blue as an inhibitor of superoxide generation by xanthine oxidase. A potential new drug for the attenuation of ischemia/reperfusion injury. Biochem Pharmacol. 1991(Jul 15); 42(3):499-506.

4. Yildiz, H., A.S. Durmus, and H. Simsek. Surgery-induced changes in red blood cell and plasma lipid peroxidation, enzymatic and non-enzymatic antioxidants, and blood hematology of female rats: protective role of methylene blue and vitamin E. Eur J Obstet Gynecol Reprod Biol. 2011(Mar); 155(1):89-93.

5. Jang, D.H., L.S. Nelson, and R.S. Hoffman. Methylene blue for distributive shock: a potential new use of an old antidote. J Med Toxicol. 2013(Sep); 9(3):242-249.

6. Valentine, E., et al. Clinical update in liver transplantation. J Cardiothorac Vasc Anesth. 2013(Aug); 27(4):809-815.

7. Legault, J., et al. Low-concentration methylene blue maintains energy production and strongly improves survival of Leigh syndrome French Canadian skin fibroblasts. J Pharm Pharm Sci. 2011; 14(3):438-449.

Acknowledgment: We would like to thank the ISHRS for this research grant funded in October 2014.

\section{Elus}

Instruments, Inc.

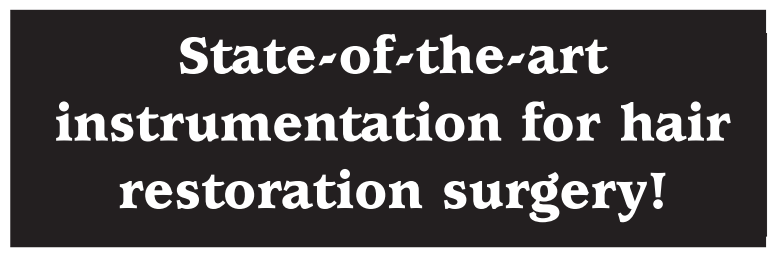

For more information, contact:

21 Cook Avenue
Madison, New Jersey 07940 USA

Phone: 800-218-9082 • 973-593-9222

Fax: 973-593-9277

Email: ellisinstruments.cellis@gmail.com www.ellisinstruments.com 